\section{$\underset{\substack{\text { hommes } \\ \text { \& migrations }}}{ }$}

\section{Hommes \& migrations}

Revue française de référence sur les dynamiques

migratoires

\section{6-1287 | 2010}

Les migrations subsahariennes

\title{
Migration des étudiants sénégalais
}

Impact sur le développement de leur pays d'origine

\section{Magatte Fall}

\section{Q OpenEdition}

1 Journals

\section{Édition électronique}

URL : http://journals.openedition.org/hommesmigrations/1755

DOI : 10.4000/hommesmigrations. 1755

ISSN : 2262-3353

Éditeur

Musée national de l'histoire de l'immigration

\section{Édition imprimée}

Date de publication : 1 juillet 2010

Pagination : 222-233

ISSN : 1142-852X

\section{Référence électronique}

Magatte Fall, « Migration des étudiants sénégalais», Hommes \& migrations [En ligne],

1286-1287 | 2010, mis en ligne le 29 mai 2013, consulté le 19 avril 2019. URL : http:// journals.openedition.org/hommesmigrations/1755; DOI : 10.4000/hommesmigrations.1755 


\section{Migration des étudiants sénégalais Impact sur le développement de leur pays d’origine}

Par Magatte Fall, docteur en géographie (Ph.D.), membre du réseau REDTAC (Im) migration du Cérium de l'université de Montréal

Le phénomène de l'exode des étudiants prend des proportions alarmantes dans les pays en voie de développement et en particulier au Sénégal. Ceci est d'autant plus préoccupant qu'il touche des secteurs et des activités de haute technologie à forte valeur ajoutée. Chaque année, des centaines d'étudiants sénégalais, titulaires de bourses subventionnées par l'État, partent étudier à l'étranger. Peu d'entre eux reviennent à la fin de leurs études, mais leurs transferts de capitaux concourent au développement économique de leur pays d'origine. 
L'expression "fuite des cerveaux", apparue dans les années cinquante, est souvent associée à l'idée de perte pour les pays de départ. Or le phénomène de la fuite des cerveaux n'est pas récent : sans le départ vers l'ouest de penseurs et d'artistes grecs à la suite du déclin de Byzance, jamais sans doute la Renaissance n'aurait-elle vu le jour. Aujourd'hui dans le monde du savoir, même si l'aller simple est toujours la règle, la mondialisation a quasiment banalisé les flux temporaires de main-d'cuvre. Les "échanges de compétences, ou de cerveaux" permettent au pays de départ tout comme aux pays d'accueil de profiter de l'expérience spécialisée acquise par des professionnels expatriés, et pas seulement de leurs envois de fonds, aussi importants soient-ils. Ainsi dans le cas de la migration des étudiants sénégalais en France faut-il parler de "fuite des cerveaux" ou faut-il adopter le concept de "la circulation des compétences ?".

Il est important de souligner que, depuis son indépendance, le Sénégal considère son système éducatif comme le secteur le plus fondamental pour son développement, en lui consacrant près du tiers de son budget. En 1998, l'État a entamé la mise en ceuvre d'un programme décennal de l'éducation et de la formation (PDEF) qui définit les nouvelles orientations du gouvernement en matière d'éducation pour les dix prochaines années. Ce programme qui inaugure une étape nouvelle dans l'évolution du système éducatif a été introduit par le gouvernement en collaboration avec les partenaires au développement, la société civile, et les acteurs du système de l'éducation pour impulser un développement quantitatif et qualitatif du système éducatif. Les objectifs majeurs définis par les différents niveaux d'enseignement sont les suivants: la généralisation de l'enseignement primaire en l'an 2010, la "réallocation" de $49 \%$ du budget de l'éducation nationale à l'enseignement élémentaire, un accès plus important d'élèves à l'enseignement moyen et secondaire, l'amélioration de la qualité de l'enseignement et la recherche universitaire.

L'espace migratoire des étudiants sénégalais est aussi varié que celui des migrants non qualifiés. En ce qui concerne les flux dans le continent européen, l'analyse du tableau no 1 ci-dessous permet d'avoir une meilleure compréhension de ce phénomène de la migration estudiantine. Elle montre l'importance de la présence des étudiants sénégalais en Europe particulièrement en Europe de l'Ouest.

\section{Des liens historiques avec la France}

Nous constatons que la France est le premier pays d'accueil de ces étudiants avec une présence de 8329 étudiants en 2004, suivie par l'Allemagne, la Suisse, la Belgique, l'Italie, le Royaume-Uni, et l'Espagne. Pays colonisateur, la France avait favorisé la formation de l'élite sénégalaise sur son territoire en octroyant des bourses d'études aux habitants des quatre communes du Sénégal, Dakar, Gorée, Saint-Louis et Rufisque. 


\section{Tableau 1. Flux des étudiants sénégalais \\ en mobilité internationale dans le monde entre 1999 et 2004 (dans 25 pays d'Europe)}

\begin{tabular}{|c|c|c|c|c|c|c|}
\hline Pays d'accueil & 1999 & 2000 & 2001 & 2002 & 2003 & 2004 \\
\hline Allemagne & 219 & 237 & 247 & 238 & 243 & 256 \\
\hline Autriche & 9 & 10 & 6 & 1 & 2 & 4 \\
\hline Belgique & 104 & 127 & 125 & 141 & 102 & 103 \\
\hline Chypre & I & I & 1 & 1 & 1 & I \\
\hline Danemark & I & 1 & 1 & I & 2 & 1 \\
\hline Espagne & 12 & 17 & 8 & 6 & 14 & 4 \\
\hline Finlande & 4 & 3 & 2 & 1 & 1 & 1 \\
\hline France & 3545 & 4079 & 5114 & 6123 & 7978 & 8329 \\
\hline Irlande & I & I & 1 & I & I & I \\
\hline Italie & 32 & 26 & 32 & 25 & 40 & 53 \\
\hline Liechtenstein & I & I & I & I & I & 2 \\
\hline Lituanie & I & I & I & I & I & 1 \\
\hline Moldavie & I & I & 6 & 3 & 1 & I \\
\hline Norvège & 3 & 2 & 3 & 4 & 5 & 3 \\
\hline Pays-Bas & 2 & 1 & 2 & 4 & 3 & 4 \\
\hline Pologne & 3 & I & I & I & I & 1 \\
\hline Portugal & I & I & I & 1 & 3 & 3 \\
\hline Roumanie & 2 & I & I & 1 & 2 & 2 \\
\hline Royaume-Uni & 25 & 22 & 26 & 26 & 30 & 39 \\
\hline Saint-Siège & 7 & I & I & I & I & I \\
\hline Slovaquie & I & I & I & 3 & I & I \\
\hline Suède & 2 & 2 & 4 & 6 & 5 & I \\
\hline Suisse & 74 & 20 & 120 & 150 & 221 & 224 \\
\hline Tchèque (république) & 4 & 6 & 6 & 3 & 2 & 2 \\
\hline Turquie & 5 & 6 & 7 & 6 & 5 & 4 \\
\hline
\end{tabular}

Source : Annuaire statistique de l'Unesco, 2004

/ : Données manquantes 
De l'indépendance à aujourd'hui, la France reste une destination toujours prisée. À partir de 1998, les effectifs des étudiants sénégalais qui partent poursuivre leurs études en France sont en pleine augmentation. Cette croissance s'explique par la volonté de la France d'assouplir l'entrée et le séjour des étudiants étrangers sur son territoire national. En effet, l'ampleur politique de la mobilité internationale des étudiants a longtemps prévalu dans les échanges entre les États. L'enjeu principal était alors de participer à l'accroissement de l'influence de la France sur la scène internationale en accueillant les futures élites des pays avec lesquels des liens privilégiés étaient entretenus.

Il convient de rappeler que l'effectif des étudiants étrangers dans les universités (y compris IUT) a connu une régression de 11,4 \% entre 1990 et 1998, passant de 136 306 à 122 142. Ils ne représentaient plus alors que 8,6 \% des étudiants inscrits contre $11,5 \%$ en 1990.

À la suite des mesures incitatives prises par les pouvoirs publics en 1998 pour améliorer l'attractivité de la France, la tendance s'est inversée. Le rapport du ministère de l'Éducation nationale, de l'enseignement supérieur et de la recherche, de juin 2005 , sur les conditions d'inscription et d'accueil des étudiants étrangers dans les universités montre que les effectifs ont augmenté régulièrement et de façon significative pour atteindre le nombre de 200588 étudiants en 2003-2004 et représenter 13,7 \% des effectifs étudiants, avec une croissance de plus de 64 \% depuis 1998.

Au-delà des seules universités, cette évolution touche l'ensemble de l'enseignement supérieur. Les étudiants étrangers qui étaient 149295 en 1998 sont 245298 en 2003-2004, soit une augmentation de plus de 64\%. En 2002, 72 \% des étudiants étrangers, soit 144423 étudiants, sont donc des étudiants en mobilité, ils étaient 57 \% en 1998, soit 69621 étudiants. Ce sont 74802 étudiants supplémentaires que les universités ont inscrits entre 1998 et 2003, soit $95 \%$ de l'augmentation des étudiants étrangers enregistrée. Pendant la même période, les universités ont inscrit 72809 étudiants français (et assimilés) de moins, d'après le même rapport.

\section{Le choix déterminant du pays de formation}

D'après nos enquêtes de terrains menées pendant notre thèse de doctorat de géographie sur la migration des étudiants sénégalais en France, ses déterminants, leurs conditions de séjour et leur devenir, leurs arrivées sont motivées et encouragées par la présence sur place de parents ou d'amis, d'où l'importance de l'appartenance ethnique - car il y a certaines ethnies qui sont spécialisées dans la migration vers la France -, sans oublier l'importance de l'origine géographique. En effet, on a une 
majorité d'étudiants citadins c'est-à-dire venus des grandes villes du Sénégal, en particulier de Dakar où il existe une classe dominante (politique, économique) qui envoie ses enfants en France. Mais les vraies motivations souvent évoquées par les étudiants sont : la recherche de la qualité des études, un diplôme reconnu sur le plan local et international pour trouver plus facilement un emploi, l'offre de bourses, la connaissance de la langue, les conditions ou facilités favorisant l'accès des étrangers à l'enseignement supérieur, la gratuité des études par comparaison avec les pays anglosaxons, la connaissance et l'image positive du pays d'accueil. Il faut également souligner d'autres facteurs que sont : la mauvaise qualité de l'enseignement supérieur du pays de départ, la défaillance de l'offre de formation dans une discipline ou un niveau donné, les conseils des parents, des amis, des situations particulières comme les grèves qui surviennent chaque année dans l'enseignement supérieur et qui peuvent durer trois mois voire plus. Ainsi, deux années ont été invalidées au Sénégal pour des raisons de longs mouvements de grèves. Il s'agit de 1988 et 1994, qui ont entrainé le redoublement des étudiants dans tous les cycles de l'enseignement supérieur.

Rappelons que le nombre de garçons est largement supérieur à celui des filles. Le faible accès de ces dernières à l'enseignement, notamment à l'université, s'explique par le poids de la tradition qui cantonne la femme à son rôle d'épouse et de mère. Voilà en somme les décisions qui conduisent à choisir la France comme pays de formation.

\section{Les dynamiques migratoires des étudiants sénégalais dans le monde}

Concernant les pays comme la Suisse, la Belgique, elles sont surtout liées au caractère francophone de ces pays. La langue ne constituant pas une barrière pour les études, l'adaptation des étudiants sénégalais dans ces universités est facilitée. Par contre la présence des étudiants sénégalais dans la plupart des autres pays tels que le RoyaumeUni, l'Espagne, l'Allemagne, l'Italie, le Portugal, est surtout liée à la coopération que ces pays entretiennent avec le Sénégal, en accordant un certain nombre de bourses dans leurs universités, pour des étudiants sénégalais qui ont déjà effectué des études de langues dans les universités du Sénégal.

Pour les pays d'Europe de l'Est, cette présence est aussi due à la coopération avec le Sénégal, à travers l'octroi d'un certain nombre de bourses d'études, notamment dans le domaine des sciences et de la technologie. C'est le cas des pays comme la République tchèque, la Slovaquie, la Lituanie, la Roumanie. 
Tableau 2. Flux des étudiants sénégalais

en mobilité internationale dans le monde entre 1999

et 2004 (dans 7 pays d'Asie)

\begin{tabular}{|l|r|r|r|r|r|r|}
\hline Pays d'accueil & 1999 & 2000 & 2001 & 2002 & 2003 & 2004 \\
\hline Arabie Saoudite & 68 & 77 & $/$ & $/$ & 86 & 61 \\
\hline Corée du Sud & $/$ & $/$ & $/$ & $/$ & $/$ & 1 \\
Inde & $/$ & 3 & 1 & $/$ & $/$ & $/$ \\
Iran & 1 & $/$ & 1 & $/$ & $/$ & $/$ \\
Japon & 16 & 20 & 24 & 25 & 24 & 27 \\
Jordanie & $/$ & 4 & $/$ & $/$ & 5 & 6 \\
\hline Malaisie & 6 & $/$ & $/$ & 5 & 5 & $/$ \\
\hline
\end{tabular}

Source : Annuaire statistique de l'Unesco, 2004 /: Données manquantes

Contrairement à l'Europe, la présence des étudiants sénégalais dans le continent asiatique est moins importante, quand on regarde le tableau n ${ }^{\circ} 2$. Nous constatons que cette présence est plus régulière au Japon. Cela est lié à l'attribution de bourses d'études par le gouvernement du Japon à des étudiants sénégalais et à des ingénieurs installés au Sénégal en vue d'un perfectionnement dans le domaine des sciences et technologies.

Par contre, en Arabie Saoudite cette présence est surtout liée à une mobilité d'étudiants issus des écoles d'enseignement de la langue arabe et de la religion musulmane à travers le Sénégal, bénéficiant de bourses pour étudier la langue arabe ou l'enseignement du Coran et des préceptes de la religion. C'est aussi le cas des étudiants qui partent étudier en Iran ou en Jordanie.

L'analyse du tableau no 3 révèle la présence de quelques étudiants sénégalais en Amérique latine notamment au Brésil et à Cuba. Cette présence estudiantine dans ces pays est liée, comme pour certains autres pays que nous avons vus plus haut, à l'octroi de bourses d'études pour un an. Permettant un approfondissement des connaissances dans un domaine plus pointu, comme les sciences et la technologie. Par contre, dans la partie de l'Amérique du Nord, cette présence est plus significative. Cela s'explique par le fait qu'ils bénéficient d'une exonération des droits d'inscription dans les universités canadiennes. Du coup la plupart des étudiants qui 
Tableau 3. Flux des étudiants sénégalais

en mobilité internationale dans le monde entre 1999 et 2004 (dans 4 Pays du continent américain)

\begin{tabular}{|l|r|r|r|r|r|r|}
\hline Pays d'accueil & 1999 & 2000 & 2001 & 2002 & 2003 & 2004 \\
\hline Brésil & $/$ & $/$ & $/$ & 1 & 4 & $/$ \\
\hline Canada & 219 & 246 & $/$ & 319 & $/$ & $/$ \\
\hline Cuba & $/$ & $/$ & $/$ & 12 & 2 & 6 \\
\hline États-Unis & 529 & $/$ & 635 & 809 & 774 & 805 \\
\hline
\end{tabular}

Source : Annuaire statistique de l'Unesco, 2004

/: Données manquantes

\section{Tableau 4. Flux des étudiants sénégalais \\ en mobilité dans le monde entre 1999 et 2004 (dans 7 pays d'Afrique noire et du Maghreb)}

\begin{tabular}{|l|r|r|r|r|r|r|}
\hline Pays d'accueil & 1999 & 2000 & 2001 & 2002 & 2003 & 2004 \\
\hline Cameroun & $/$ & $/$ & $/$ & $/$ & $/$ & 1 \\
Congo & $/$ & $/$ & $/$ & $/$ & $/$ & 6 \\
Gabon & 11 & $/$ & $/$ & $/$ & $/$ & $/$ \\
Gambie & $/$ & 11 & $/$ & $/$ & $/$ & $/$ \\
Maroc & 141 & 172 & 227 & $/$ & 364 & 435 \\
Mauritanie & $/$ & $/$ & $/$ & $/$ & 86 & 61 \\
\hline Togo & 1 & 1 & $/$ & $/$ & $/$ & $/$ \\
\hline
\end{tabular}

Source : Annuaire statistique de l'Unesco, 2004 /: Données manquantes

y sont inscrits appartiennent à l'élite dominante, car les frais de séjour et d'études sont très dispendieux, les étudiants ne bénéficiaient pas de la possibilité de trouver un emploi pour financer leurs études jusqu'en 2006, date à laquelle cette contrainte a été levée par les autorités canadiennes.

Cette présence d'étudiants sénégalais est également en augmentation aux États-Unis. L'obtention d'un visa d'études pour les étudiants sénégalais inscrits dans les universités 
américaines est en effet facilitée. Par ailleurs on constate une prolifération d'agences d'aide à la recherche d'une préinscription dans les universités américaines.

Dans une interview accordée au journal quotidien Le Soleil, madame Beth A. Payne, consul américain au Sénégal, souligne que "les étudiants sont prioritaires sur les visas ${ }^{(1)}$ ". D'où une augmentation considérable du nombre de visas accordés aux étudiants sénégalais.

Concernant les flux d'étudiants sénégalais dans le continent africain, d'après le tableau no 4, ils sont relativement plus nombreux au Maghreb en particulier au Maroc que dans les autres pays de l'Afrique noire. Cela est surtout marqué par la coopération entre l'État du Sénégal et le Maroc qui envoient chaque année quelques centaines d'étudiants dans leurs universités respectives selon un accord qui date de 1963. Il faut ajouter que le royaume chérifien accorde un nombre important de bourses d'études pour les étudiants sénégalais qui sont inscrits dans ses universités.

\section{Des migrations subventionnées par l'État sénégalais}

Au Sénégal, cette nouvelle forme d'émigration concerne surtout les lauréats du “concours ", organisé pour récompenser les meilleurs élèves des lycées du Sénégal. Mais aussi des étudiants de deuxième et troisième cycle, formés dans les universités sénégalaises. Ainsi, tous les lauréats et beaucoup d'étudiants du deuxième et troisième cycle partent à l'étranger chaque année soit juste après leur formation, soit pendant leur formation. On constate de véritables brigades de recrutement des lauréats, qui sont mises en place à travers un système d'octroi de bourse pour une spécialisation dans un domaine bien précis.

Les coûts de ce phénomène sont incommensurables pour le développement économique et social du Sénégal. En l'absence de statistiques globales et fiables, on peut avancer quelques éléments d'appréciation qui donnent une idée de l'ampleur de cette forme d'émigration sur le développement.

Au niveau des coûts en formation, un étudiant boursier sénégalais en France coûte environ 3000 euros par an à l'État sénégalais. À ce montant, il faut ajouter toutes les dépenses liées à sa formation avant son départ. Cette indication permet de mesurer l'ampleur des pertes subies par l'État du Sénégal, dont le budget supporte les coûts en formation d'une partie non négligeable des ingénieurs et cadres des pays développés. Autrement dit, un volume appréciable des dépenses budgétaires relatives à l'éducation et à la formation sert en fait à financer le développement technologique des pays bénéficiaires de cette nouvelle forme d'émigration. 
Au niveau de l'appel aux experts étrangers, cette fuite de compétences s'inscrit dans la part de plus en plus importante et coûteuse donnée à l'expertise internationale. Ce phénomène grève lourdement les ressources déjà très maigres des pays en développement, dont le Sénégal. Dans ce cadre, "pour combler ces fuites, le continent fait non seulement appel au personnel qualifié en provenance des pays occidentaux (100000 expatriés non africains), mais également débourse de fortes sommes (4 milliards \$ américains annuellement $)^{2}$ ".

Ces données assez élémentaires montrent l'autre facette de cet exode en termes de coûts financiers directs et qui certainement connaittront de fortes augmentations au cours des prochaines années.

\section{Les coûts du recrutement croisé}

Dans ces conditions, les pays en développement, dont le Sénégal, se trouvent dans un véritable cercle vicieux. D'un côté, ils dépensent beaucoup d'argent pour la formation de leurs étudiants et cadres qui fuient à l'étranger, de l'autre, ils doivent faire venir des experts étrangers pour leurs besoins à des coûts exorbitants.

Enfin, les coûts en expériences sont difficilement quantifiables et sont souvent source de dégâts considérables. Il s'agit des coûts inhérents au "pompage" de cadres, ingénieurs, médecins, et enseignants-chercheurs qui ont déjà une grande expérience au sein des entreprises, universités, et hôpitaux dans le pays de départ. Il faut souligner qu'ils partent pour la majorité pour une spécialisation à l'étranger et restent pour la plupart après leur formation. Ces coûts peuvent se mesurer en termes de perte de fonctionnement rationnel et efficace, de réduction de compétitivité, de non-réalisation de projets... pour les entreprises et l'administration du Sénégal victimes de ce véritable pillage. En effet, cette dernière catégorie d'émigrés nouveaux est non seulement bien formée, mais a également une expérience pratique importante au sein du tissu économique du pays.

Ainsi d'après l'article de Dialigué Faye du journal Le Soleil: "La proportion des Sénégalais ayant reçu une éducation supérieure et qui ont émigré vers les pays développés a

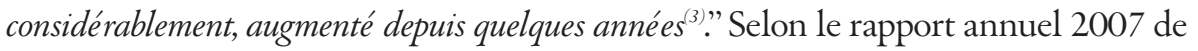
la Conférence des Nations unies sur le commerce et le développement (Cnuced) présenté par Pierre Encontre, chef économiste au bureau de Genève en Suisse, le taux du "stock du capital humain" vivant à l'extérieur du Sénégal était évalué à $11 \%$ en 1990. Dix ans après, en 2000, ce taux est passé à $24 \%$. Il y aurait à peu près 24000 diplômés de l'enseignement supérieur qui sont à l'extérieur du Sénégal. Selon M. Encontre, "sous l'angle de la balance des paiements, il y a certainement des avantages du 
point de vue macroéconomique". Ainsi, il voulait montrer que les apports des transferts d'argent ainsi effectués par ces migrants peuvent s'avérer bénéfiques pour l'économie du pays. Mais "c'est en termes de matière grise nécessaire pour développer un pays que cela est assez problématique". Car, pour lui, "une fuite des cerveaux est un facteur qui empêche une économie d'être dynamique. C'est quelque chose de dramatique $e^{(4) "}$.

\section{Des réformes globales pour retenir les cadres sénégalais}

Face à l'ampleur du phénomène de l'exode des compétences et de ses conséquences très fâcheuses, il est devenu de plus en plus urgent d'arrêter cette saignée ou du moins d'en atténuer les dommages. Ainsi, une véritable stratégie nationale visant à retenir les cadres, ingénieurs, médecins et à faire rentrer le maximum d'entre eux doit être initiée et mise en ceuvre. Celle-ci doit s'articuler autour d'un ensemble de mesures et d'actions cohérentes et complémentaires visant à créer un environnement général favorable à ces compétences. Parmi les mesures de réformes à envisager, on peut insister, en particulier, sur la nécessité d'améliorer en profondeur les mécanismes de fonctionnement de l'État et des entreprises à travers l'installation d'une véritable méritocratie : respect des compétences réelles, véritables plans de carrière, ouvrir 
la voie de la responsabilité aux jeunes. Il s'agit de moraliser la vie publique et au sein des entreprises au moyen de l'application des principes de priorité, d'intégrité, d'honneur, de moderniser les mentalités (culture du travail, sens du professionnalisme) tout en élargissant les espaces de liberté et de démocratie. Il est nécessaire, également, de mettre en application une véritable politique de promotion de la recherche. Il s'agit entre autres de développer les centres et instituts de recherche tout en les dotant des moyens humains et techniques satisfaisants, d'élargir l'interface entre le monde de la recherche et celui des affaires...

\section{De la "fuite des cerveaux" à la "circulation des compétences"}

En somme, nous pouvons dire que la "fuite des cerveaux" est devenue la nouvelle forme d'émigration des Temps modernes. Le Sénégal n'échappe pas à ce phénomène qui prend une ampleur sans précédent et très inquiétante. Ainsi, une grande partie des étudiants sénégalais formés au Sénégal partent chaque année à l'étranger et restent dans les pays développés à la fin de leurs études. De même que ceux qui ont gagné de l'expérience dans les entreprises sénégalaises et qui partent pour une spécialisation ne reviennent plus. Ces situations entraînent des pertes aussi bien en termes de coûts financiers, qu'en termes d'expériences et de performances des entreprises et des administrations.

Mais, aujourd'hui, il faut être beaucoup plus nuancé quand on parle "de fuite de cerveaux" en évoquant la notion de perte ou de gain. Pour faire face à l'avancée de la science et de la technologie, le Sénégal est obligé comme les autres pays en voie de développement, d'envoyer ou de laisser partir ses étudiants et intellectuels étudier ou se former à l'étranger, en particulier dans les pays développés. Ainsi, il convient de repenser la façon dont la fuite des compétences est analysée et perçue, l'idée serait de laisser tomber le concept négatif de "fuite des cerveaux" pour parler de "circulation des cerveaux". Car, jusqu'en 1990, cette expression de "fuite des cerveaux" évoquait l'idée d'une migration définitive et à sens unique de personnes hautement qualifiées, venant du monde en voie de développement vers les pays industrialisés.

De nos jours, ce type de migration n'est plus un déplacement définitif dans un seul sens, les effets positifs de la migration sur le progrès économique et social et culturel ont fini par faire comprendre que la circulation des compétences et de la maind'ceuvre pouvait être un catalyseur du développement. On peut remarquer de multiples retombées globales de ce type de migration internationale : la création et le transfert de connaissance, la constitution d'une main-d'ceuvre instruite et 
qualifiée et le développement des relations commerciales sont dans certaines mesures partagés par les pays situés aux deux extrémités de la chaîne migratoire, par le biais des étudiants qui retournent dans le pays d'origine.

\section{Les expatriés, une ressource indispensable pour le Sénégal}

De nombreux expatriés contribuent déjà massivement à l'économie de leur pays de départ par les transferts de fonds à destination de leur famille, notamment les diplômés qui restent dans le pays de formation ou dans un autre pays du Nord. Ainsi, pour appuyer cette thèse, une étude de la DPEE (Direction de la prévision et des études économiques), citée par l'APS (Agence de presse sénégalaise), révèle que les transferts de fonds des Sénégalais de l'extérieur ont représenté en 2007 environ 460 milliards de FCFA, soit trois fois plus que les investissements directs étrangers. Toujours selon la même source, la principale conclusion tirée de cette étude mentionne que les envois de fonds des migrants réduisent significativement le nombre de ménages en dessous du seuil de pauvreté, (incidence) à hauteur de $31 \%$. Ce qui signifie que le tiers des ménages recevant des transferts auraient été pauvres s'ils ne recevaient pas ces fonds. Cette étude révèle que les transferts de fonds accroissent en moyenne de 60 \% les dépenses par tête des ménages qui les reçoivent, même si la répartition selon les milieux de résidence et le niveau de revenu fait sortir des disparités au sein des ménages. Le découpage des ménages en cinq groupes de revenu allant du plus pauvre au plus riche (quintile) montre une évolution croissante de l'effet des transferts en fonction du revenu.

Cette catégorie de personnes qualifiées n'est pas perdue pour le Sénégal et constitue une réserve de main-d'ceuvre qualifiée que le pays pourrait utiliser en cas de décollage économique. En effet, pendant la période de l'alternance en 2000 au Sénégal, le pouvoir politique avait fait venir de l'étranger des personnes qui occupaient de hautes fonctions dans les pays développés pour des postes ministériels. Cependant, cette idée novatrice a subitement été freinée par une décision qui a repositionné au sommet de l'État des militants de partis politiques, ce qui demeure un préjudice pour une meilleure "circulation des compétences".

\section{Notes}

1. Quotidien Le Soleil, juin 2007.

2. Quotidien Le Soleil, "Fuite des cerveaux en Afrique : Une saignée de 23000 universitaires", 23 octobre 2007.

3. Quotidien Le Soleil, "Nations unies, 10 ans de fuite de cerveaux : Le Sénégal a perdu 24 \% de ses diplômés

du supérieur", 21 juin 2007.

4. Quotidien Le Soleil, idem. 